\title{
Morphology Study and Planning of the Active Distribution Network in the Energy Internet
}

\author{
Xuan ZHOU ${ }^{1}$, Zhuo $\mathrm{LI}^{1}$, Chong $\mathrm{CHEN}^{1, \mathrm{a}}$, Fei-Peng $\mathrm{YANG}^{1}$, Xue-Song \\ $\mathrm{ZHANG}^{2, \mathrm{~b}}$ and Chen $\mathrm{XU}^{2}$ \\ ${ }^{1}$ State Key Laboratory of Advanced Electromagnetic Engineering and Technology, Huazhong
University of Science and Technology, Wuhan, Hubei, China
}

${ }^{2}$ SG ZJEPC Electric Power Research Institute Hangzhou, Zhejiang, China

a772188846@qq.combee_zxs@163.com

\begin{abstract}
Keywords: Energy internet, Planning of the distribution network, Energy exchanger, Energy router, Multi-energy interface.
\end{abstract}

\begin{abstract}
Energy internet, first proposed by American economist Rifkin, is recognized as the trend of the future energy industry, and there is no doubt that the largest energy network at present- power system will play a significant role in it. This paper firstly analyses the novel demands of future distribution network orienting to energy internet. To satisfy the special demands, the future distribution network will develop to be a system with the function of multi-energy conversion and utilization. On this base, the framework of the future distribution network is proposed involving supply and demand of different types of energy. Then the energy flow of the system is analyzed and the optimal planning model of the distribution network, including the cost structure and the operation constraints, is built. In the end, the planning model is simulated using multi-objective particle swarm optimization (MOPSO) algorithm. The simulation result verifies the effectiveness of the proposed model.
\end{abstract}

\section{Introduction}

With the drying up of traditional fossil energy and the environment problem it causes, seeking a new way of energy structure and supply has gained more and more attention. Energy internet, a new energy system combined with advanced energy technology and information technology, which is first proposed by American economist Rifkin, is considered to be the trend of the future energy industry development [1, 2]. Structurally, energy internet is a blend of electricity, gas, cooling, heating, transportation, and even more different forms of energy network as well as the information network; Functionally, with the help of the advanced information and computing technology, energy internet makes the coordinated operation and complementarity of large area, multi-energy systems possible, achieving better economic and environmental benefits. At present, there is still no final conclusion about the concrete morphology of energy internet, but considering the convenience of transmission, utilization and conversion of electric energy, power grid will play a main role in the future energy internet, and distribution network will also develop from a pure electric power distribution network to a new type of power distribution network with the function of multi-energy (electricity, gas, cooling, heating and so on) conversion, substitution and utilization.

Researchers have studied the morphology of energy internet from different perspective. Literature [3] initially defines energy internet and expounds the basic architecture. Literature [4] refers to the worldwide energy internet research, practice work and analyzes the technology connotation of energy Internet. Literature [5] builds the unified network topology model of energy internet based on the forefront complex network modeling method. Literature [6] describes the basic idea of the framework construction and the top-level design of energy internet, and discusses its development path. Considering the basic needs of energy internet, the morphological structure and basic function of future distribution network will change accordingly, but there is little prospective study at present. Literature [7] proposes a utility framework of urban energy internet 
and analyzes the integration of supply \& consumption of the future distribution network. Literature [8] proposes the concept of integrated distribution system orienting to energy internet and expounds its morphology systematically, including structure, equipment, operation control and industry morphologies. Research on distribution network orienting to energy internet pays more attention to the basic morphology and key technologies and rarely involves its planning, only planning of electricity, gas integration system reported [9-10].

This paper analyzes the new demands of distribution network in the energy internet scenario. On this base, the new framework of distribution network adapted to the development of energy internet is proposed, with its key equipment and functions expounded. Based on the model of multi-energy conversion and utilization in future distribution network, the optimal planning model of the distribution network is built, and it's verified by simulation using multi-objective particle swarm optimization (MOPSO) algorithm.

\section{Framework of Active Distribution Network}

Considering the core and the branch status of power system in energy internet, the future distribution network should accomplish higher power distribution demands compared with traditional distribution network, and have the new capabilities serving energy internet on the other hand. The former involves greater power supply ability, higher transfer efficiency and reliability, greater ability of load acceptance (especially for new type of load in the energy internet scenario) and so on. The latter involves conversion interface for different forms of energy, network topology widely connected with information network, new control morphology and control strategy for "source-network-load-storage" coordination, multi-energy coordination, multi-demand-response coordination and so on.

To satisfy the new requirements mentioned above, the future distribution network should be a generalized distribution system that is power-grid-led and has the function of multi-energy (electricity, gas, cooling, heating and so on) conversion, substitution and utilization, the basic framework is shown in Fig. 1, and the power flow in Fig. 2, in which different energy networks are equivalent to energy buses.

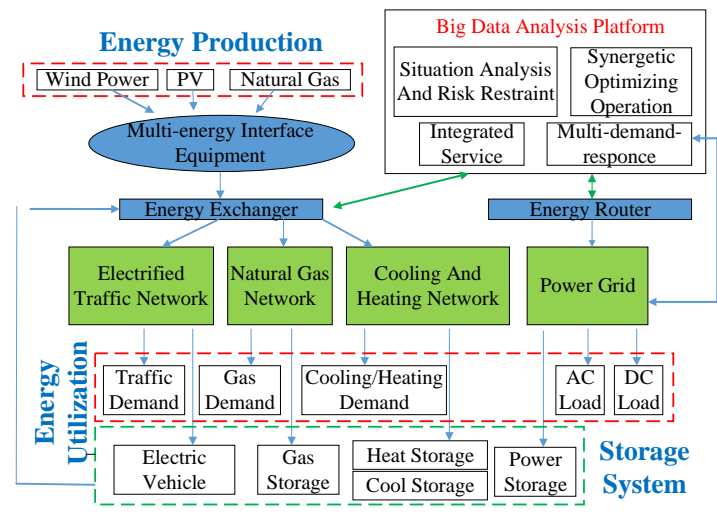

Fig. 1 Basic framework of future active distribution network 


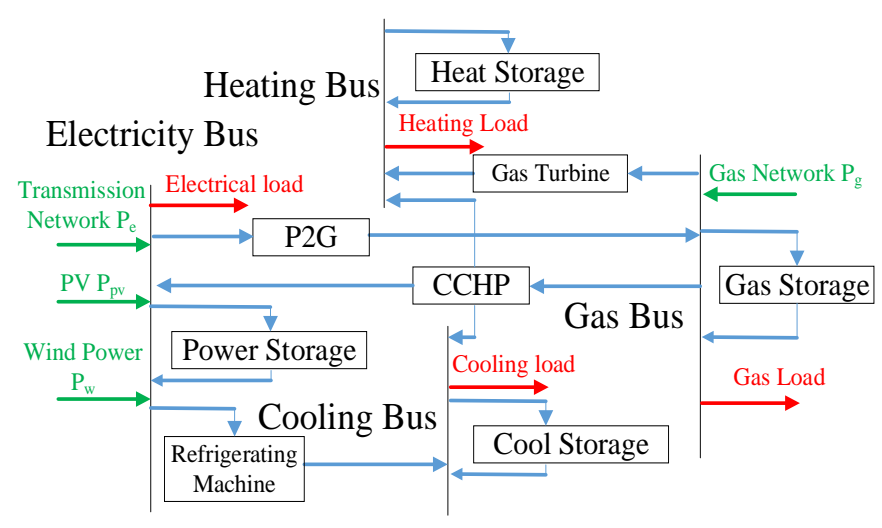

Fig. 2 Energy flow of future distribution network

In the active distribution network, the relevant nomenclatures are as follow: PV-photovoltaic; CCHP- combined cooling heating and power; AC Load- alternating current load; DC Load- direct current load.

1) Multi-energy interface equipment: the key equipment that makes multi-energy (electricity, gas, cooling, heating and so on) conversion possible, including electric vehicle (electricity and traffic), gas turbine (gas and electricity), combined cooling heating and power (CCHP, electricity, gas, cooling and heating) and so on. The equipment should be plug-and-play, to satisfy the requirement of optimal configuration of different types of energy.

2) Energy exchanger: the key equipment that implements the function of multi-energy distribution. Energy exchanger is a device of physic and information fusion, and its input is the information of multi-energy demand and supply. With the help of multi-energy interface equipment, it converts a definite proportion of energy (input, supply) to a suitable proportion (output, demand), and then transfers the output energy to the corresponding network, thus achieving "source-networkload-storage" coordination and multi-energy coordination.

3) Energy Router: compared with other forms of energy transmission network, the control of the power grid is more flexible but more complexes, and in the meantime it has to meet the higher demand of power distribution than traditional distribution network. Therefore the electric energy output from energy exchanger is controlled by energy router for uniform distribution. The energy router described in this article is a device of pure electric energy. Energy router in different levels is responsible for the tasks in the corresponding areas, such as the power distribution, power quality control, information system control and maintenance, etc. As a key equipment in the future power grid, energy router improves the control characteristic of the traditional distribution network, which can accomplish higher power distribution demands.

4) Big data analysis platform: with the diversification of the energy form, the access of new intelligent devices and the increasing demand of controllability and observability, the scale and complexity of the data in energy internet will significantly increase compared with the traditional distribution network. It includes system data such as automation of the network, equipment data such as terminal information collection equipment and energy conversion equipment, macroeconomic data, environment data and so on. The huge amounts of data should to be uniformly managed. As the control center of the future distribution system, big data analytics platform is the data collection point and is the key point for the system security, stability and economic operation.

\section{Multi Objective Optimization Planning Model}

This article is aimed at the planning of the capacity of the multi-energy interface equipment and energy storage, achieving the optimal economy when satisfying the demand of different load. 


\section{The Objective Function}

There are two objective functions in the planning model: minimum cost and load shedding, as in

$$
\begin{aligned}
& \min C=C_{1}+C_{2}+C_{3}-C_{4} \\
& \min \Delta P_{\text {load }}=\Delta P_{\text {load }}^{e}+\Delta P_{\text {load }}^{g}+\Delta P_{\text {load }}^{h}+\Delta P_{\text {load }}^{c}
\end{aligned}
$$

Where $C_{1}$ is the investment cost; $C_{2}$ is the alternative cost; $C_{3}$ is the operation and maintenance cost; $C_{4}$ is the remanent value of equipment; $\Delta P_{\text {load }}^{e}, \Delta P_{\text {load }}^{g}, \Delta P_{\text {load }}^{h}, \Delta P_{\text {load }}^{c}$ are the load shedding of electricity, gas, heating and cooling respectively.

1) The investment cost. The Capital Recovery Factor (CRF) represents the ratio of equivalent annual cost to the initial investment cost of the device:

$$
C R F(L, N, r)=\frac{r(1+r)^{N}}{(1+r)^{L}-1} \quad r=\frac{i-f}{1+f}
$$

Where $\mathrm{N}$ is the project cycle ( $\mathrm{N}=20$ years in this article); $\mathrm{L}$ is the equipment life; $\mathrm{r}$ is the annual interest rate, $\mathrm{i}$ is the nominal loan rate more than 5 years, $\mathrm{f}$ is the rate of inflation. Thus the investment cost

$$
C_{1}=\sum_{i} N_{i} \cdot C_{i} \cdot C R F\left(L_{i}, N, r\right)
$$

where $N_{i}$ represents the number of the devices; $C_{i}$ represents the initial investment cost of the devices; $i \in\{P 2 G, e 2 c, e s, C C H P, B, g s, h s, c s\}$ represents $\mathrm{P} 2 \mathrm{G}$, refrigerating machine, power storage, CCHP, gas turbine, gas storage, heat storage and cool storage respectively.

2) The alternative cost. $L_{\text {rep } i}$ represents if the equipment should be substituted when $L_{i} \neq N$ and the working life of the equipment when substituted. If $L_{\text {rep } i}=0$, the equipment doesn't need to be substituted, as in

$$
L_{r e p, i}=L_{i} \cdot \operatorname{INT}\left(\frac{N}{L_{i}}\right)
$$

The alternative cost of equipment $i$

$$
C_{21}=N_{i} C_{i} f_{r, i} \operatorname{SFF}\left(L_{i}, r\right)
$$

Where

$$
f_{r, i}=\left\{\begin{array}{ll}
\frac{C R F(N, N, r)}{C R F\left(\mathrm{~L}_{r e p, i}, N, r\right)} & L_{r e p, i}>0 \\
0 & L_{r e p, i}=0
\end{array} \quad \operatorname{SFF}\left(L_{i}, r\right)=\frac{r}{(1+r)^{L_{i}}-1}\right.
$$

Because the life of storage depends on the operation mode and the status of charging and discharging, the coefficient of losses $\alpha$ is introduced to represent the equivalent annual cost of the storage. Thus, the alternative cost

$$
C_{2}=C_{21}+C_{22}=\sum N_{i} C_{i} f_{r, i} \operatorname{SFF}\left(L_{i}, r\right)+\alpha N_{i} C_{i}
$$


3) The operation and maintenance cost. The operation cost mainly includes the cost of gas and electricity. The operation and maintenance cost is calculated as equation (8):

$$
C_{3}=\sum_{i} N_{i} C_{M, i}+\sum_{t=1}^{T}(\beta E(t)+\gamma G(t))
$$

Where $\mathrm{CM} i$ is the operation cost of the equipment; $\beta$ is the price of electricity; $\mathrm{E}(\mathrm{t})$ is the electricity consumption in one year; $\gamma$ is the price of gas; $E(t)$ is the gas consumption in one year.

4) The remnant value of equipment. The remanent value of equipment is calculated as equation (9):

$$
C_{4}=\sum_{i} C_{r e m, i}=\sum_{i} N_{i} C_{i} L_{r e m, i} / L_{i} \cdot \operatorname{SFF}(r, \mathrm{~N})
$$

Where $L_{r e m, i}=L_{i}+L_{r e p, i}-N$ is the residual life of the equipment.

\section{The Restraint Conditions}

1) The power restraint conditions. The electricity and gas transferred from the upper level of the network are limited, thus the lower limit and upper limit of gas and electric power are included in the model.

2) The power balance. The power balance for different energy buses is shown in equations (10)(13).

$$
\begin{aligned}
& P_{e}+P_{P V}+P_{W}+P_{e s, d}+P_{C C H P}^{e}=P_{\text {load }}^{e}+P_{e s, c}+P_{p 2 g}^{e}+P_{e 2 c}^{e}-\Delta P_{\text {load }}^{e} \\
& P_{g}+P_{g s, d}+P_{e 2 g}=P_{\text {load }}^{g}+P_{c C H P}^{g}+P_{g 2 h}^{g}+P_{g s, c}-\Delta P_{\text {load }}^{g} \\
& P_{h s, d}+P_{C C H P}^{h}+P_{g 2 h}^{h}=P_{\text {load }}^{h}+P_{h s, c}-\Delta P_{\text {load }}^{h} \\
& P_{c C H P}^{c}+P_{e 2 c}^{c}+P_{c s, d}=P_{\text {load }}^{c}+P_{c s, c}-\Delta P_{\text {load }}^{c}
\end{aligned}
$$

where $P_{e}, P_{P V}, P_{w}$ are the input electric power from transmission network, distributed PV power and wind power; $P_{g}$ is the input gas power; $P_{\text {load }}^{e}, P_{\text {load }}^{g}, P_{\text {load }}^{h}, P_{\text {load }}^{c}$ are the electricity, gas, heating and cooling load; $P_{e s, d}, P_{g s, d}, P_{h s, d}, P_{c s, d}$ are the discharge power of power storage, gas storage, heat storage and cool storage; $P_{e s, c}, P_{g s, c}, P_{h s, c}, P_{c s, c}$ are the charge power of power storage, gas storage, heat storage and cool storage; $P_{C C H P}^{g}, P_{C C H P}^{e}, P_{C H P}^{h}, P_{C C H P}^{c}$ are the input gas power and the output electric, heating and cooling power of CCHP; $P_{p 2 g}^{e}, P_{p 2 g}^{g}$ are the input electric power and output gas power of P2G; $P_{e 2 c}^{e}, P_{e 2 c}^{c}$ are the input electric power and output cooling power of refrigerating machine; $P_{g 2 h}^{g}, P_{g 2 h}^{h}$ are the input gas power and output heating power of gas turbine.

3) The operational constraints of the multi-energy interface equipment. This part takes CCHP as an example, as in equations (14)-(15). 


$$
\begin{aligned}
& P_{C C H P}^{e}=\sum_{i=1}^{N_{C C H P}} P_{C C H P}^{i} \eta_{g 2 e}, P_{C C H P}^{h}=\sum_{i=1}^{N_{C C H P}} P_{C C H P}^{i} \eta_{g 2 h}, P_{C C H P}^{c}=\sum_{i=1}^{N_{C C H P}} P_{C C H P}^{i} \eta_{g 2 c} \\
& P_{C C H P, \min }^{i} \leq P_{C C H P}^{i} \leq P_{C C H P, \max }^{i}
\end{aligned}
$$

Where $P_{C C H P}^{i}$ is the input gas power of CCHP i; $\eta_{g 2 e}, \eta_{g 2 h}, \eta_{g 2 c}$ are the conversion efficiency of electricity, heating and cooling; $P_{C C H P, \max }^{i} P_{C C H P, \min }^{i}$ are the upper limit and lower limit of the input power of CCHP.

4) The operational constraints of the storage system. This part takes power storage as an example. There is upper limit for the charging power and discharging power, upper limit and lower limit for the SOC. The SOC is calculated as equations (16).

$$
\operatorname{SOC}_{t+1}^{i}=\operatorname{SOC}_{t}^{i}\left(1-\sigma_{e s}^{i}\right)+\left(u_{c}^{i} P_{e s, c}^{i} \eta_{e s, c}^{i}-u_{d}^{i} P_{e s, d}^{i} / \eta_{e s, d}^{i}\right) \Delta t, 0 \leq u_{c}^{i}+u_{d}^{i} \leq 1
$$

Where $\operatorname{SOC}_{t}^{i}$ is the SOC of the power storage at time $\mathrm{t} ; \eta_{e s, c}^{i}, \eta_{e s, c}^{i}$ are the charging efficiency and discharging efficiency; $u_{c}^{i}, u_{d}^{i}$ are the state of charging/discharging, 1 for charging/discharging and 0 for non- charging/discharging.

\section{Exponential Analysis}

In this part, the planning model described above is solved using MOPSO, with the different types of load, the load characteristic, the input, conversion, storage and output of the energy considered. The load curve is shown in Fig. 3-6. For easy analysis, all the dimension of power is kilowatt.

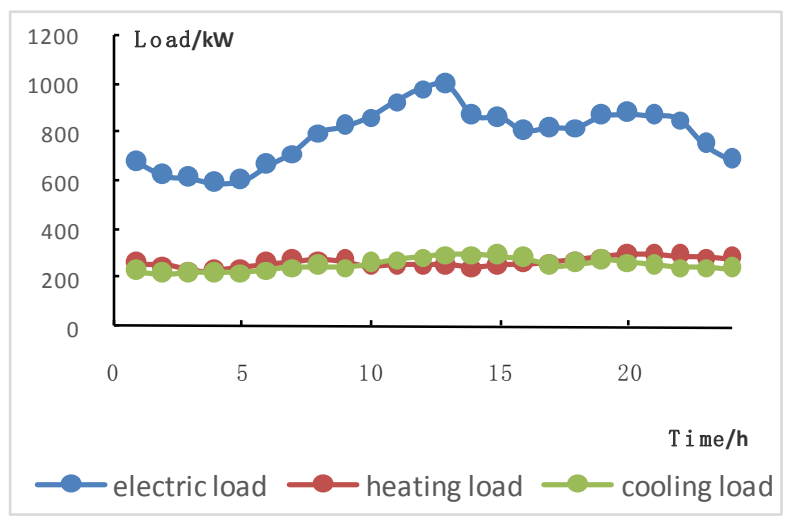

Fig.3 The electrical, heating, cooling load of spring and autumn 


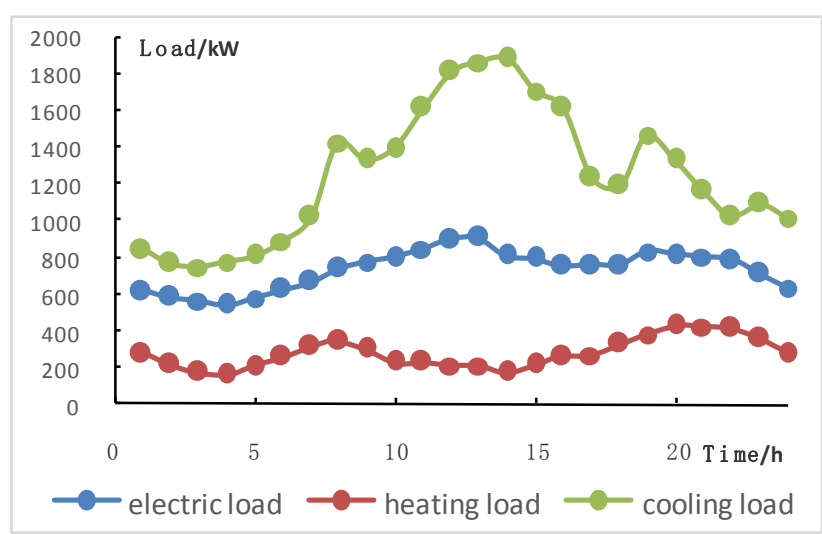

Fig.4 The electrical, heating, cooling load of summer

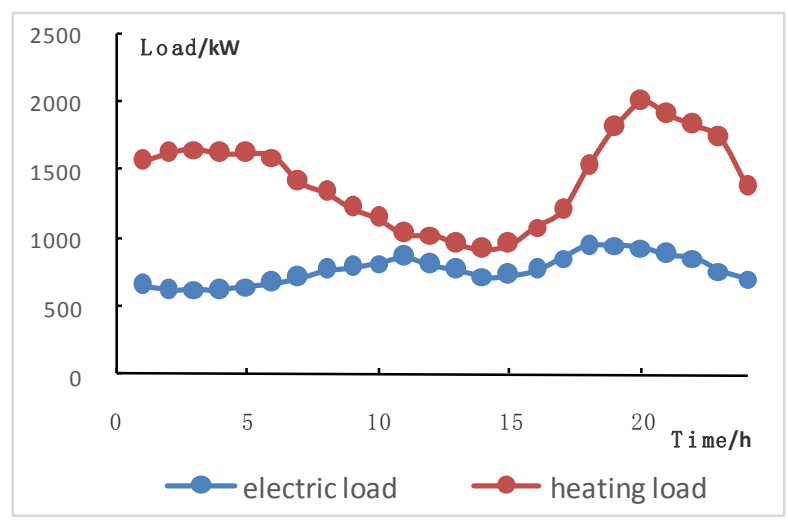

Fig.5 The electrical, heating, cooling load of winter

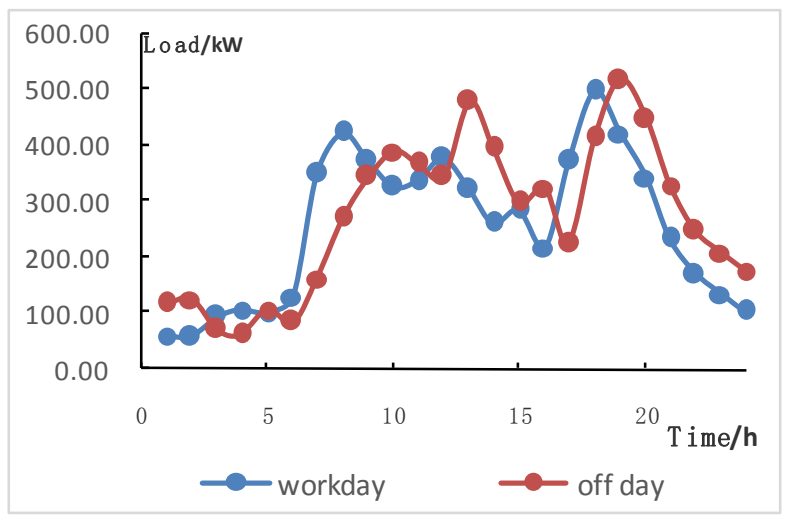

Fig.6 The diagram of gas load

The input of future distribution network includes electricity from transmission network, distributed PV and wind power and the gas from the gas network. The basic parameters of the planning equipment are shown in TABLE I.

The result of the simulation is shown in Fig. 7. The load shedding is less than $0.8 \%$, satisfying the operation requirements of the system. The results of minimum cost and load shedding are shown in TABLE II. Comparing the two conditions, to lower the load shedding, the number of storage increases, because the energy conversion equipment is enough to supply most of the load, and only a few storages are needed when the load is high. Considering the mainstream position of electricity, 
the number of power storage increases the most. In addition, as shown in Fig. 7, with the decline of load shedding, the ascending range of cost is different, For example, at the point 1, 2, 3, the increase of cost makes no difference to the load shedding. When planning in practice, the overall consideration of economy and reliability is necessary.

Table.1 Equipment parameter

\begin{tabular}{clll}
\hline Equipment & $\begin{array}{l}\text { The investment cost/ } \\
\text { thousand dollars }\end{array}$ & Life/year & Capacity/kW \\
\hline P2G & 710.75 & 8 & 1000 \\
$\begin{array}{l}\text { Refrigerating } \\
\text { machine }\end{array}$ & 148.07 & 15 & 1000 \\
Power storage & 0.21 & 5 & 12 \\
CCHP & 266.53 & 15 & 1000 \\
Gas turbine & 1066.13 & 10 & 1000 \\
Gas storage & 0.74 & 20 & 100 \\
Heat storage & 5.18 & 15 & 120 \\
Cool storage & 1.48 & 15 & 120 \\
\hline
\end{tabular}


Table.2The planning results of minimum cost and minimum load shedding rate

Equipment

Result of minimum cost

\section{Result of minimum load} shedding

$\mathrm{P} 2 \mathrm{G}$

Refrigerating machine

Power storage

CCHP

Gas turbine

Gas storage

Heat storage

Cool storage
2

1

3

2

1

1

12

9
1

1

15

2

1

28

2

11

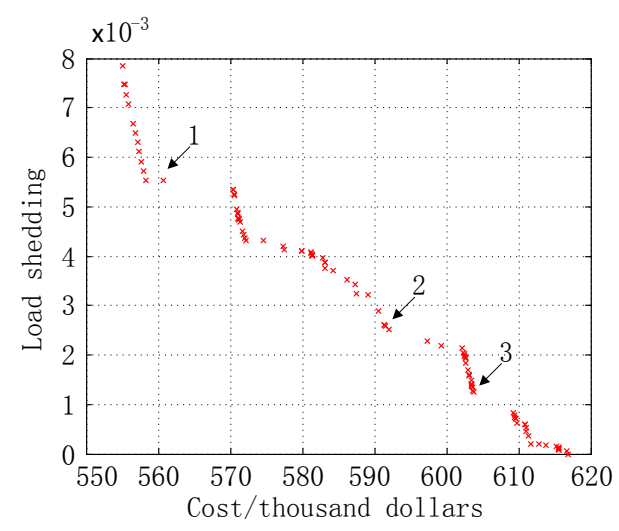

Fig. 7 The simulation result of the model

\section{Conclusions}

This article is aimed at the development of energy internet, analyzing the new demands of future distribution network in the energy internet scenario. On this base, the framework of future distribution network is proposed, involving supply and demand of different types of energy. Based on the construction and operation model of future distribution network, the optimal planning model is built, including the cost structure, the energy conversion and the load supply. In the end, the planning model is simulated using MOPSO. The result shows that the new type of distribution network can satisfy the load demand of different kinds through the conversion of energy. Within the 
constraints of economy and reliability, the planning results are between the minimum cost and the minimum load shedding. The best result in a certain planning scenario can be decided through the overall consideration of economy and reliability.

\section{Acknowledgement}

This work is partially supported by the National Natural Science Foundation of China (51537003), and in part by State Grid Zhejiang Electric Power Company.

\section{References}

[1] Bolla R, Bruschi R, Davoli F, et al. Energy efficiency in the future internet: a survey of existing approaches and trends in energy-aware fixed network infrastructures [J]. Communications Surveys \& Tutorials, IEEE, 2011, 13(2): 223-244.

[2] Tsoukalas L H, Gao R. From smart grids to an energy internet: Assumptions, architectures and requirements[C]. Third International Conference on Electric Utility Deregulation and Restructuring and Power Technologies, 2008. Nanjing: IEEE, 2008: 94-98.

[3] DONG Zhaoyang, ZHAO Junhua, WEN Fushuan, et al. From smart grid to Energy Internet: basic concept and research framework [J]. Automation of Electric Power Systems, 2014, 38(15): 111. (In Chinese)

[4] Tian Shiming, Luan Wen peng, Zhang Dong xia, et al. Technical forms and key technologies on energy internet [J]. Proceedings of the CSEE, 2015, 35(14): 3482-3494. (In Chinese)

[5] Cai Wei, Zhao Hai, Wang Jinfa, et al. A unifying network topological model of the energy internet macro-scope structure [J]. Proceedings of the CSEE, 2015, 35(14): 3503-3510. (In Chinese)

[6] LIU Kaijun. Development path exploration of energy internet [J]. Electric Power Construction, 2015, 36(10): 5-10. (In Chinese)

[7] Pu Tianjiao, Liu Kewen, Chen Naishi, et al. Design of ADN based urban energy internet architecture and its technological issues [J]. Proceedings of the CSEE, 2015, 35(14): 3511-3521. (In Chinese)

[8] Liu Dichen, Peng Sicheng, Liao Qingfen, et al. Outlook of future integrated distribution system morphology orienting to energy internet [J]. Power System Technology, 2015, 39(11): 3023-3034. (In Chinese)

[9] Unsihuay-Vila C, Marangon-Lima J W, de Souza A C Z, et al. A model to long-term, multiarea, multistage and integrated expansion planning of electricity and natural gas systems [J]. IEEE Transactions on Power Systems, 2010, 25(2): 1154-1168.

[10] Saldarriaga C A, Hincapié R A, Salazar H. A holistic approach for planning natural gas and electricity distribution networks [J]. IEEE transactions on power systems, 2013, 28(4): 4052-4063. 